\title{
Scanning Hall probe microscopy of unconventional vortex patterns in the two-gap $\mathrm{MgB}_{2}$ superconductor
}

\author{
J. Gutierrez, ${ }^{1, *}$ B. Raes, ${ }^{1}$ A. V. Silhanek,,${ }^{1,2}$ L. J. Li, ${ }^{1}$ N. D. Zhigadlo, ${ }^{3}$ J. Karpinski, ${ }^{3}$ J. Tempere, ${ }^{4}$ and V. V. Moshchalkov ${ }^{1}$ \\ ${ }^{1}$ INPAC-Institute for Nanoscale Physics and Chemestry, Katholieke Universiteit Leuven, Celestijnenlaan 200 D, B-3001 Leuven, Belgium \\ ${ }^{2}$ Département de Physique, Université de Liège, B- 4000 Liège, Belgium \\ ${ }^{3}$ Laboratory for Solid State Physics, ETH Zürich, 8093-Zurich, Switzerland \\ ${ }^{4}$ University of Antwerp, TQC, B-2610 Antwerp, Belgium \\ (Received 8 February 2012; published 22 March 2012)
}

\begin{abstract}
The low magnetic field vortex patterns nucleation and evolution in a high-quality two-gap superconductor $\mathrm{MgB}_{2}$ single crystal have been investigated by low-temperature scanning Hall probe microscopy. Large areas have been imaged with single-vortex resolution while changing systematically the thermodynamic parameters for field and temperature. The obtained patterns have been studied and compared with those of a reference $2 \mathrm{H}-\mathrm{NbSe}_{2}$ single crystal. We found that the observed vortex patterns in $\mathrm{MgB}_{2}$ (e.g., stripes, clusters) appear due to competing vortex-vortex interactions as suggested by the theory of type- 1.5 superconductivity.
\end{abstract}

DOI: 10.1103/PhysRevB.85.094511 PACS number(s): 75.70.Kw, 71.45.-d, 74.70.Ad, 89.75.Kd

\section{INTRODUCTION}

One of the most striking signatures of self-organization is spontaneous pattern formation. Among the morphologies observed, stripes are very fascinating objects. Stripe formation occurs in a wide variety of physical and chemical systems, ${ }^{1}$ which range in diversity from a type- 1 superconducting film to Langmuir monolayers, magnetic films, lipid monolayers, liquid crystals, polymer films, and to chemical mixtures displaying complex steady-state reaction-diffusion (Turing) patterns. These stripe patterns are often stabilized by competing interactions between the particles. ${ }^{1}$ For soft condensed matter, such pattern formation can occur when the particles experience intermediate-range repulsion and short-range attraction, such as in certain types of colloidal systems. ${ }^{2}$ In addition to stripe phases, numerous other patterns can appear as a function of density, temperature, or particle interaction strength, including bubble, clump, and uniform crystalline phases. ${ }^{1}$ Strikingly, it has been recently shown that in a widely investigated two-gap superconductor as $\mathrm{MgB}_{2}$, superconducting vortices accommodate themselves forming striped flux patterns. ${ }^{3}$ Since this peculiar vortex clustering shared reminiscence with both type- 1 and type- 2 superconductors, it was coined as type-1.5 superconductivity. At the core of the dichotomy between the two types of superconductivity lies the concept of interface energy separating a superconducting $(\mathrm{S})$ domain from a normal (N) region, first introduced by London ${ }^{4}$ and later calculated by Ginzburg and Landau (GL). ${ }^{5}$ For $\kappa<1 / \sqrt{ } 2$ (type-1), the S-N wall energy is positive, and the magnetic field is expelled from the bulk; whereas for $\kappa>1 / \sqrt{ } 2$, the S-N wall energy is negative, and the superconductor gets rapidly flooded with tiny tubes of magnetic field carrying one unit of flux quantum.

Recently, this classification of superconductors has been challenged by Babaev and Speight $^{6}$ when considering a multicomponent superconductor, i.e. consisting of two coupled condensates. In that paper, the authors propose that, in these materials, the flux distributes unevenly, combining bundles of vortices, as in type-2 materials, separated by vortex-free regions, as in type-1 superconductors, due to competing vortex-vortex long-range attraction and short-range repulsion. Vortex states similar to those predicted theoretically were first experimentally observed in 2009 by Moshchalkov et al. $^{3}$ in clean prototypical two-band superconductor $\mathrm{MgB}_{2}$. In these experiments, inhomogeneous vortex patterns, such as stripes and clusters, were found at low fields by Bitter decoration technique. However, the Bitter decoration technique suffers from certain drawbacks, such as the ex situ determination of the vortex patterns and the fact that only poor temperature control at the actual moment of decorating the vortex lattice is typically achieved. Later on, scanning SQUID microscopy experiments ${ }^{7}$ performed on similar clean $\mathrm{MgB}_{2}$ crystals revealed, at very low magnetic fields, unusual vortex patterns typical of a type-1.5 superconductor.

In this paper, we investigate vortex patterns in clean $\mathrm{MgB}_{2}$ single crystals by using scanning Hall probe microscopy (SHPM) with single-vortex resolution and offering the possibility to change the thermodynamic variables, magnetic field and temperature, while simultaneously visualizing the vortex patterns. We have been able to observe the progressive formation of stripes, investigate the reproducibility and stability of different vortex patterns, and shake the vortices. In addition, we have carried out a back-to-back comparison with the reference conventional type-2 superconductor $\mathrm{NbSe}_{2}$. These studies provide direct information about the vortex pattern formation and evolution as well as the contribution of pinning and surface barriers to the vortex stripe phase stabilization in type-1.5 superconductors.

\section{EXPERIMENTAL DETAILS}

The $\mathrm{MgB}_{2}$ single crystals have been grown by a hightemperature and high-pressure cubic anvil technique, using a precursor containing $\mathrm{Mg}, \mathrm{B}$, and $\mathrm{BN}$ as described in Ref. 8. The quality of similar crystals has been already confirmed by crystallographic studies ${ }^{9}$ and magnetization measurements showing low pinning and clean limit behavior. ${ }^{10}$ Additionally, $2 \mathrm{H}-\mathrm{NbSe}_{2}$ crystals grown by the standard iodine vapor transport method ${ }^{11}$ have been used in this work as a reference well-understood type- 2 superconductor.

The SHPM images were obtained by using a modified lowtemperature scanning Hall microscope from Nanomagnetics 
Instruments. The typical scanning area of a single image ranges between $14 \times 14$ (at $4 \mathrm{~K}$ ) to $24 \times 24$ (at $30 \mathrm{~K}$ ) $\mu \mathrm{m}^{2}$. The Hall sensor is kept at about 500-700 $\mathrm{nm}$ above the surface of the sample, giving rise to a maximum signal of $\sim 2 \mathrm{G}$ at the core of a single quantized vortex, whereas the field resolution of the Hall cross is better than $0.1 \mathrm{G}$. By locating the Hall cross just above the surface of the crystals, we are able to determine the normal-to-superconducting phase transition temperature as the temperature at which the out-of-phase component $\chi^{\prime \prime}$ of the ac response has a maximum. Using a magnetic field amplitude of $1 \mathrm{Oe}$ and an excitation frequency of $77 \mathrm{~Hz}$, we obtain a critical temperature $T_{c}=38.2 \mathrm{~K}$ with a transition width $\delta T_{c} \approx 90 \mathrm{mK}$ for the $\mathrm{MgB}_{2}$ crystal, whereas for the $\mathrm{NbSe}_{2}$ crystal, we found a $T_{c} \approx 7 \mathrm{~K}$ with a transition width $\delta T_{c} \approx 100 \mathrm{mK}$.

\section{UNCONVENTIONAL VORTEX PATTERNS IN MgB}

In order to corroborate the presence of unconventional vortex arrays in $\mathrm{MgB}_{2}$, we have obtained SHPM images under field-cooling (FC) conditions, i.e. the sample is cooled down from $T>T_{c}$, to a chosen temperature in presence of an external field. It is well known that following this protocol guarantees a nucleated vortex state closer to the equilibrium configuration than under zero-field-cooling conditions, where surface barriers and pinning give rise to a more pronounced irreversible behavior. Figure 1 directly compares the flux distribution in the $\mathrm{NbSe}_{2}$ single crystal [Fig. 1(a) at $1 \mathrm{Oe}$ and Fig. 1(c) at $2 \mathrm{Oe}$ ] with that obtained in the $\mathrm{MgB}_{2}$ crystal
[Fig. 1(b) at 1 Oe and Fig. 1(d) at 2 Oe] after field-cooling down to $4.2 \mathrm{~K}$.

Although $\mathrm{NbSe}_{2}$ exhibits a nearly perfect triangular vortex lattice with long-range order in agreement with a scenario where pinning is weak, the $\mathrm{MgB}_{2}$ crystal shows a highly inhomogeneous vortex distribution with coexistence of vortex chains and extensive vortex-free regions. These results confirm previous studies by Bitter decoration ${ }^{3}$ and scanning SQUID microscopy ${ }^{7}$ in $\mathrm{MgB}_{2}$ single crystals from different sources. The question naturally arises whether the anomalous vortex patterns seen in $\mathrm{MgB}_{2}$ result from unconventional vortex interactions $s^{3,7,12}$ or simply reproduce an accidental peculiar inhomogeneous pinning landscape strong enough to prevent the formation of a regular Abrikosov lattice. Before addressing this point, let us first consider whether a triangular lattice as seen in $\mathrm{NbSe}_{2}$ necessarily implies negligible pinning. For the $\mathrm{NbSe}_{2}$ single crystal, the average distance between first neighbors $\left(d_{v v}\right)$ nicely follows the relation for a triangular lattice $d_{v v}=\sqrt{ }\left(1.1547 \phi_{0} / B\right)$, with $B$ (the average internal field) being replaced by $H$ (the applied external field), Fig. 2. Considering the penetration of the magnetic field in our sample $H_{p}(4.2 \mathrm{~K})=H_{c 1}(4.2 \mathrm{~K}) \sqrt{ } d / W \sim 36$ Oe (here, $H_{c 1}$ is the lower critical field, $W$ the sample half width, and $d$ its thickness ${ }^{13}$ ), it is surprising that the above relation for $d_{v v}(B)$, with $B=H$, works perfectly well all the way down to $1 \mathrm{Oe} \ll H_{p}$. This indicates that, in $\mathrm{NbSe}_{2}$, the vortex lattice is retained in a metastable state (i.e. frozen) by surface barriers or pinning sites at high temperatures, where $H_{c 1}$ is negligible

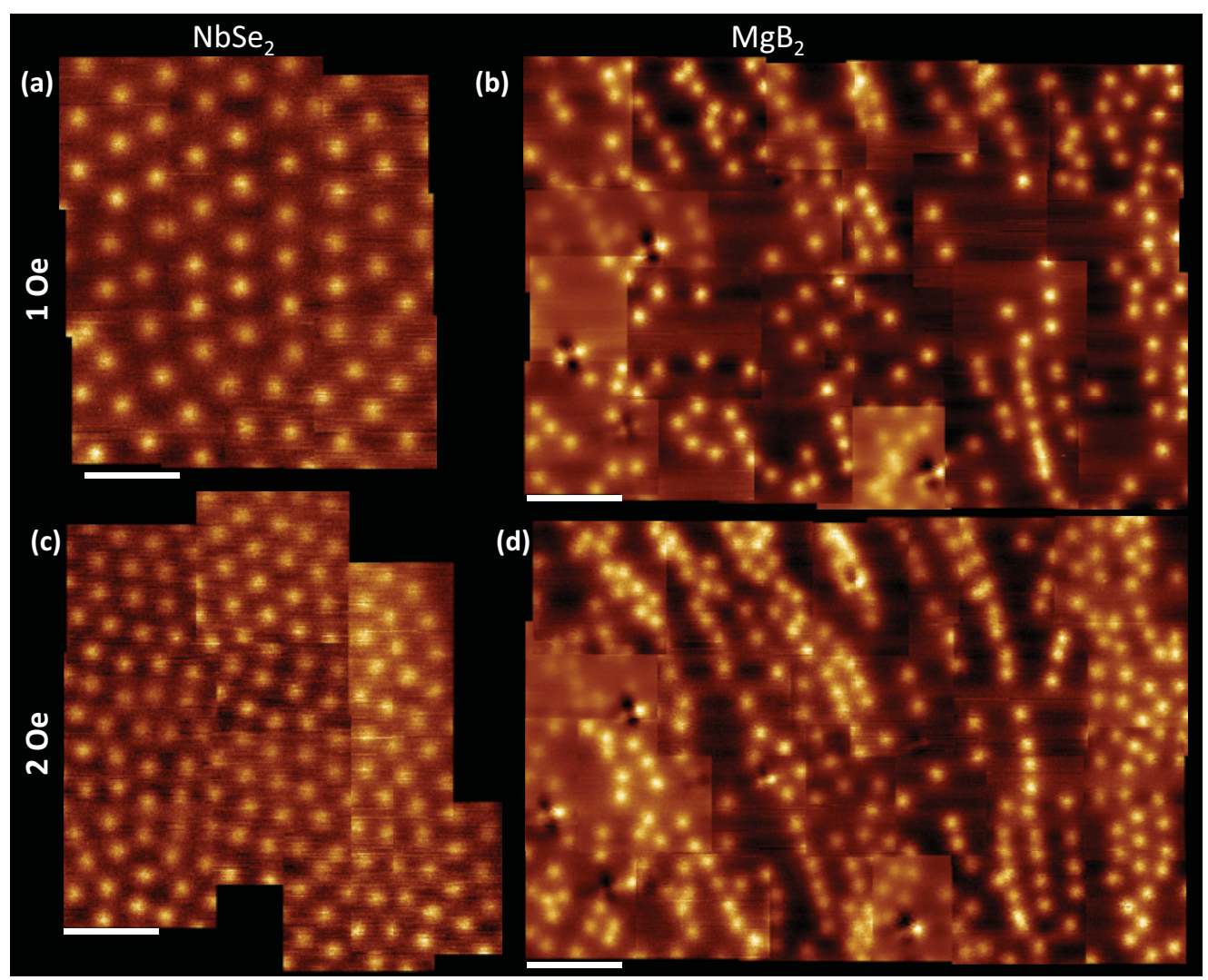

FIG. 1. (Color online) Scanning Hall probe microscopy images after performing an FC at 1 Oe for the (a) $\mathrm{NbSe}_{2}$ and (b) $\mathrm{MgB}_{2} \mathrm{single}$ crystals; and after doing FC at 2 Oe for (c) $\mathrm{NbSe}_{2}$ and (d) $\mathrm{MgB}_{2}$ single crystals. Images are taken at $4.2 \mathrm{~K}$. The white bar on each picture corresponds to a length of $10 \mu \mathrm{m}$. 


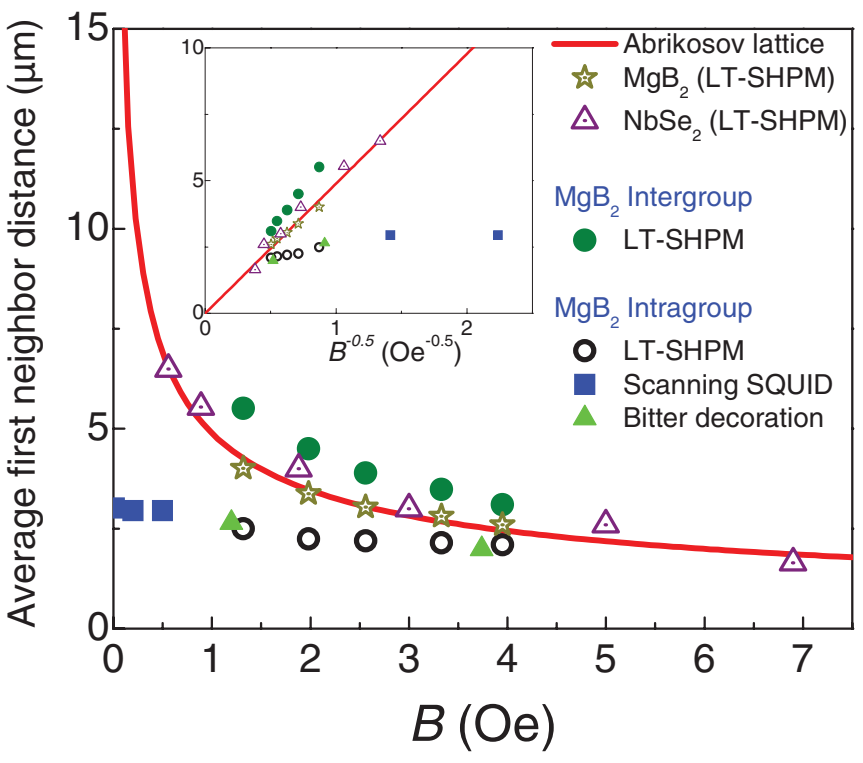

FIG. 2. (Color online) Average first neighbors distance as a function of $B$ for an $\mathrm{NbSe}_{2}$ single crystal (open triangles), an $\mathrm{MgB}_{2}$ single crystal (open stars), and for a perfect Abrikosov lattice (solid line). Additionally, there is represented the intergroup first neighbor distances for an $\mathrm{MgB}_{2}$ single crystal (full circles) and the intragroup first neighbor distances for different $\mathrm{MgB}_{2}$ single crystals; squares, triangles, and open circles correspond to scanning Squid microscopy, Bitter decoration, and to our SHPM results, respectively. The inset shows the same data as a function of $1 / \operatorname{sqrt}(B)$.

and $B \sim H$. In other words, the temperature at which the vortex pattern is formed $T_{q}$ seems to be substantially higher than the actual temperature of the experiment. ${ }^{14,15}$ We can identify this freezing temperature as the temperature above which the vortex motion prevents a clear identification of vortices by SHPM and the image looses all contrast. Following this criterion, we have found that, for $\mathrm{NbSe}_{2}, T_{q} \geqslant 0.978 T_{c}$, in agreement with previous experiments, ${ }^{14}$ while for the $\mathrm{MgB}_{2}$, $T_{q} \geqslant 0.967 T_{c}$.

Figures 1(c) and 1(d) show the evolution of the flux patterns for both materials exactly at the same spots as in panels (a) and (b), respectively, but at higher magnetic field, $H=2$ Oe. For the $\mathrm{NbSe}_{2}$ crystal, a vortex lattice containing some defects ${ }^{16}$ is observed. As it has been pointed out by Larkin and Ovchinnikov, any disorder in a superconductor, no matter how weak, could destroy the long-range positional order in the vortex lattice due to collective pinning. ${ }^{17}$ The increase in the magnetic field reveals that (i) the vortex stripes in $\mathrm{MgB}_{2}$ have a range of preferential directions, and (ii) they are not straight but rather curved, i.e. they cannot be related to crystallographic orientations of the atomic lattice. It is also worth noticing that some vortex-free regions at 1 Oe become occupied at $2 \mathrm{Oe}$, indicating that the voids in the vortex lattice do not appear due to repulsive pinning potentials. Furthermore, at $2 \mathrm{Oe}$, it is possible to find stripes containing two rows of vortex chains forming a zigzag structure similar to that seen in narrow superconducting ribbons with weak pinning. ${ }^{15,18}$ In the case of broader stripes, containing three or more vortex chains, vortices with sixfold coordination, as in an Abrikosov lattice, can be also found. These features strongly suggest that the vortex clustering is not a consequence of an inhomogeneous vortex pinning.

Interestingly, vortices in $\mathrm{MgB}_{2}$ not only depart dramatically from a triangular array, but they also violate locally the relation $d_{v v}=\sqrt{ }\left(1.1547 \phi_{0} / H\right)$ found for the $\mathrm{NbSe}_{2}$ single crystal. The existence of vortex clusters and stripes separated by vortex-free regions leads to a unique bimodal vortex distribution which has no counterpart in type- 2 superconductors. This bimodal behavior can be split into an intragroup and an intergroup distribution. ${ }^{3,7}$ We have found that the average first vortex neighbor distance in the intragroup distribution has changed only slightly, from $2.5 \mu \mathrm{m}$ at 1 Oe to $2.25 \mu \mathrm{m}$ at $2 \mathrm{Oe}$. An explanation of this unique behavior can be found through the combination of the long-range attractive and short-range repulsive $v-v$ interactions characteristic of the type- 1.5 regime, where the $v-v$ interaction potential, which is magnetic field independent, presents a minimum at a vortex separation $d_{\min } \cdot{ }^{12}$ Dao et al. predicted $d_{\min } \sim 2 \mu \mathrm{m}$ in $\mathrm{MgB}_{2}$ for the vortex lattice freezing at $T_{q} / T_{c}=t_{q}=0.97 .{ }^{19}$ This estimate is in very good agreement with our experimentally determined intragroup average first neighbor distance, giving further confirmation of the type-1.5 superconductivity in the studied clean $\mathrm{MgB}_{2}$ crystals.

In Fig. 2, we summarize the obtained average first neighbor vortex distances for $\mathrm{NbSe}_{2}$ and $\mathrm{MgB}_{2}$ single crystals as a function of the local induction $B$. The local induction has been calculated as the number of vortices divided by the total area of the image. It is shown that both the $\mathrm{NbSe}_{2}$ and the $\mathrm{MgB}_{2}$ follow very well the expected behavior to $B=(2 / \sqrt{ } 3) \phi_{0} / d_{v v}{ }^{2}$ (shown in the graph as the solid red/dark gray line). Nonetheless, it is worth noticing that, in the $\mathrm{MgB}_{2}$ single crystal, the average first neighbor vortex distances in the intergroup distribution show strong magnetic field dependence while the intragroup distribution $d_{\min }(B)$ data demonstrate only, as already mentioned, a weak dependence on the magnetic field. We observe that the average $v$ - $v$ intracluster distance changes only from $2.5 \mu \mathrm{m}$ at 1 Oe to $2 \mu \mathrm{m}$ at 5 Oe, in good agreement with the equilibrium distance $d_{\text {min }}$ proposed by the theory. ${ }^{12}$ Since the theory predicts that $d_{\min }$ is field independent, in our FC experiments, $d_{\text {min }}$ should vary with the magnetic field through $t_{q}(B)=T_{q} / T_{c}(B)$, which explains why a weak dependence on the magnetic field is seen. This striking feature has no counterpart in a type-2 superconductor, irrespective of the details of the pinning landscape.

\section{STRIPE PATTERN EVOLUTION IN MgB}

Since in the absence of surface barriers or no pinning at all, one could expect strong degeneracy in the stripe ground state orientation each time an FC is performed, ${ }^{20}$ data shown in Fig. 1(d) point out the possibility that pinning still might play a certain role in their stabilization. In order to address this point, we have performed successive FC experiments down to $4.2 \mathrm{~K}$, under identical conditions and in the same area for both investigated materials. In Fig. 3(a), we show four successive FCs at 0.9 Oe for $\mathrm{NbSe}_{2}$; and in Fig. 3(b), we repeat the same experiment for $\mathrm{MgB}_{2}$. The $\mathrm{NbSe}_{2}$ shows a weakly distorted triangular lattice, which at every FC nucleates somewhat at a different position and with the principal axes 


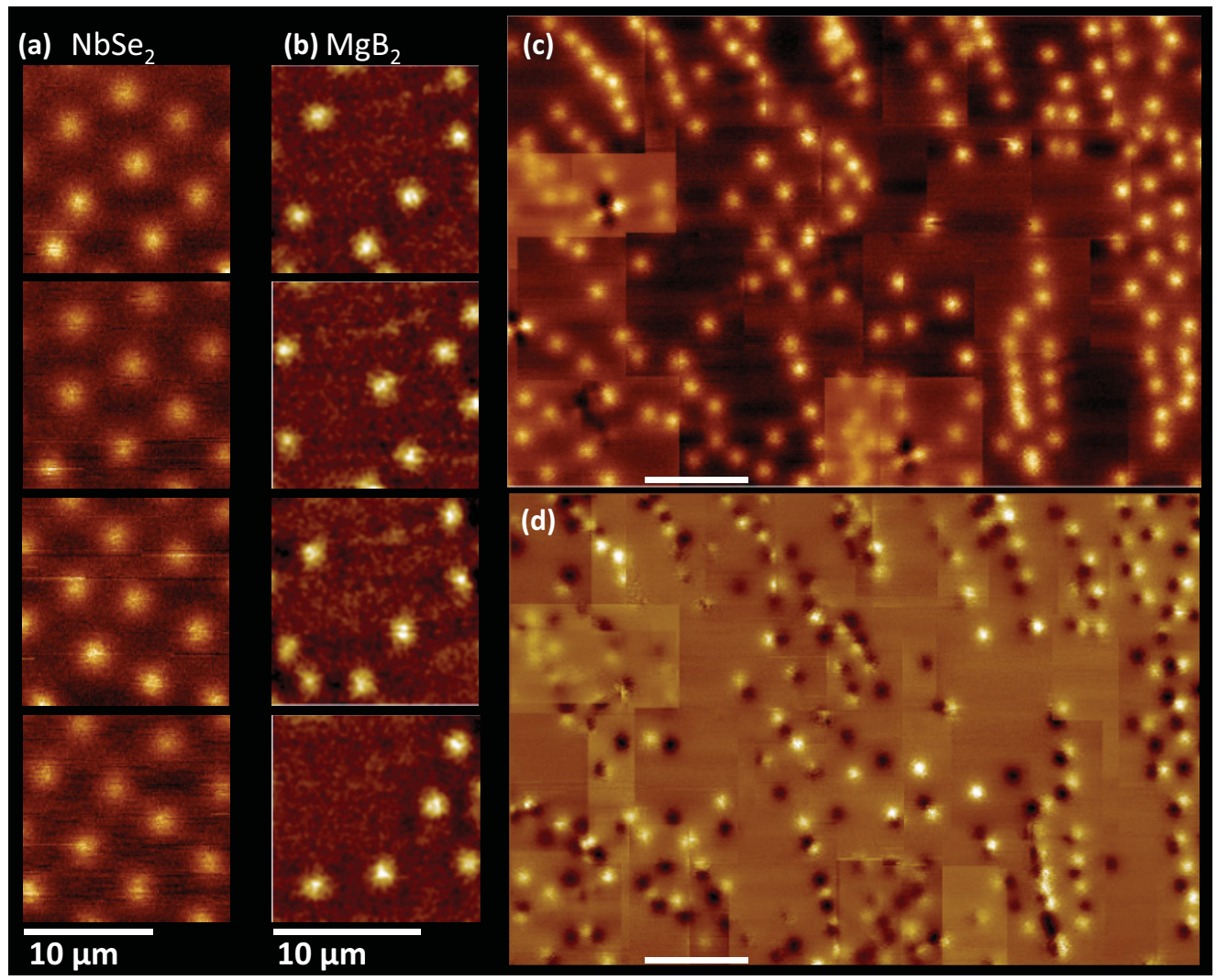

FIG. 3. (Color online) Four successive FCs at 0.9 Oe for (a) $\mathrm{NbSe}_{2}$ and (b) $\mathrm{MgB}_{2}$ single crystals. Panel (c) shows an FC at $1 \mathrm{Oe}$ for the $\mathrm{MgB}_{2}$ crystal. Panel (d) shows the difference in vortex position from two consecutive FCs. Images are taken at $4.2 \mathrm{~K}$. The white bar on each picture corresponds to a length of $10 \mu \mathrm{m}$

of the triangular array slightly rotated. The same experiment performed for the $\mathrm{MgB}_{2}$ crystal reveals a behavior similar to that for the $\mathrm{NbSe}_{2}$, but with very unusual inhomogeneous vortex patterns. Individual vortices nucleate in the successive FCs at positions that differ by more than $1 \mu \mathrm{m}$, a distance which is larger than the typical range of the elementary pinning interaction $\left(r_{p} \approx \xi\right.$ for fields $\left.B \leqslant 0.25 B_{c 2}\right)$. ${ }^{17}$ This behavior rules out the possibility of the existence of isolated strong pinning centers. Indeed, it has been demonstrated that, in the presence of such strong pinning sites, the positions of the vortices are not random, and they nucleate preferentially at these sites. ${ }^{21}$ Clearly, our experiments reveal that, in both systems, the existing pinning centers must be quite diluted and weak, in agreement with the high quality of the crystals.

Since the explored area in Fig. 3(b) (about $16 \times 16 \mu \mathrm{m}^{2}$ ) is rather small compared with the total crystal surface $(300 \times$ $200 \mu \mathrm{m}^{2}$ ), we have performed other field-cooling experiments on the $\mathrm{MgB}_{2}$ crystal at $1 \mathrm{Oe}$, covering nearly the same extended area as in Fig. 1(b) $\left(68 \times 45 \mu \mathrm{m}^{2}\right)$. The results are given in Fig. 3(c) and in the short animation presented as supplementary material (animation slide 1$)^{22}$ where we show a superposition of the two FCs. By comparing the two images, it is found that, even though generally individual vortices do not nucleate at the same position, there is a tendency for stripes and vortex-free regions to maintain their position and orientation. To better show this; in Fig. 3(d), we present the difference between two consecutive FCs [those shown in Figs. 1(b) and 3(c)]. Where a bright spot is observed, a vortex nucleated during the first FC [Fig. 1(b)], but not during the second FC [Fig. 3(c)]. Contrary to that, where a dark spot appears, a vortex nucleated during the second FC in vortex-free position seen at the first FC. It is important to mention that there exists an unavoidable error in our spatial distribution that comes mainly from the composition and alignment of the two images. We estimate this error to be certainly less than $0.5 \mu \mathrm{m}$, which in the image equals to approximately $1 / 3$ of the size of a vortex. Therefore, all bright and dark spots in Fig. 3(d) which fall below this size can actually represent vortices nucleating at the same spot.

In Fig. 4, we show two successive nucleations at 5 Oe in a partial area of the images shown in Fig. 3. At 5 Oe, the vortex-free regions have greatly shrunk in size, while vortex clusters have grown and merged with some of the stripes appearing at lower fields. It is relevant to highlight that, at the right part of both images, extended vortex-free regions still coexist with long curved stripes where vortices with sixfold symmetry can be observed. Here, both the randomness of the vortex nucleation and the preferred direction of the vortex clusters and stripes are accentuated (an animation showing the evolution of the vortex pattern for $\mathrm{MgB}_{2}$ from 1 Oe up to 5 Oe can be found as supplementary material, animation slide 2) ${ }^{23}$ It is relevant at this point to notice that the presence of any type of bias produced by the boundaries, a substrate, a small in-plane component of the magnetic field, or an external drive, such as a shear, breaks the symmetry of the 


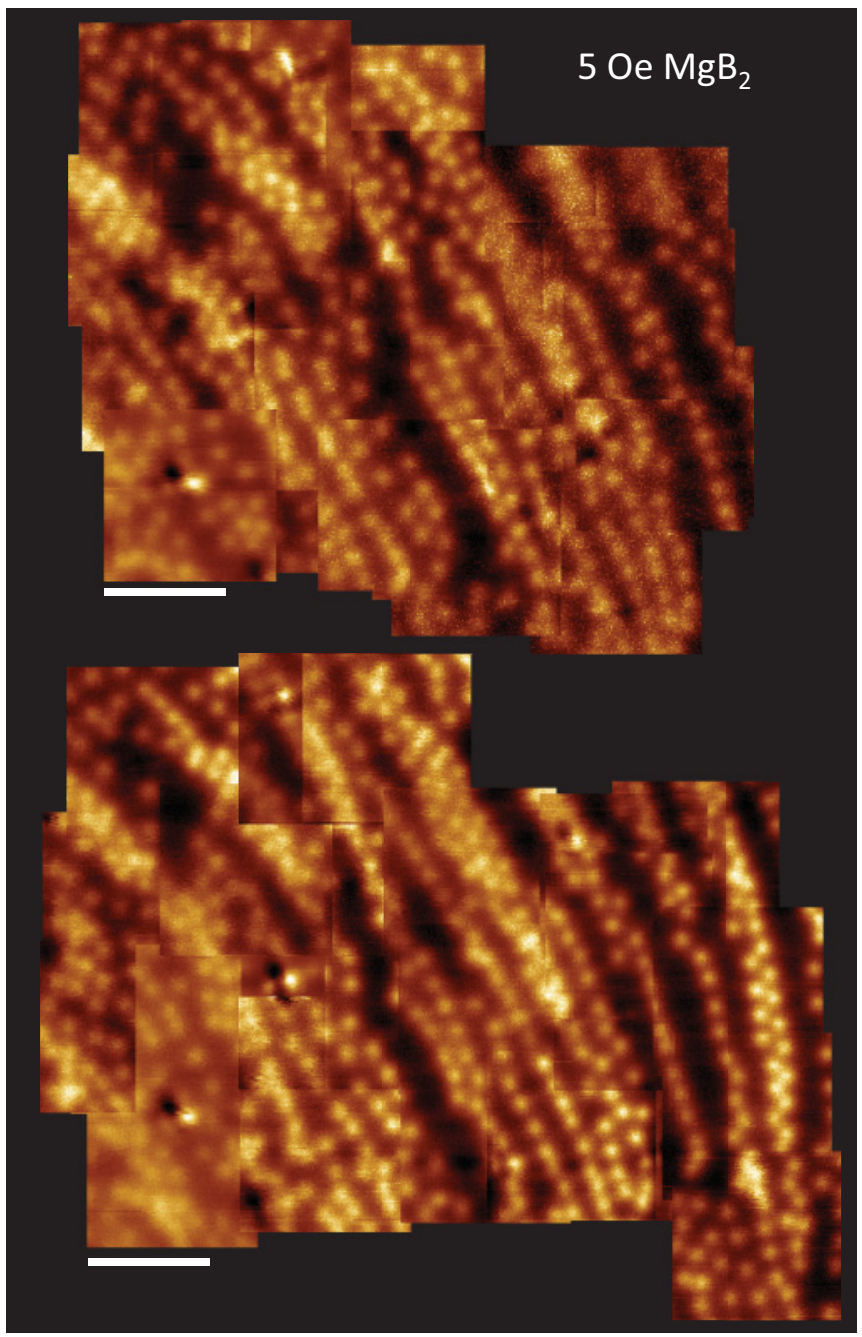

FIG. 4. (Color online) (a) Two successive field cools at 5 Oe for the $\mathrm{MgB}_{2}$. The images are taken at $4.2 \mathrm{~K}$. The white bar on each picture corresponds to a length of $10 \mu \mathrm{m}$.

stripe ground state and causes the stripes to align in a single direction. $^{20}$

\section{VORTEX PATTERN STABILITY IN MgB}

Thus far, we have shown that the $\mathrm{MgB}_{2}$ crystal exhibits a very weak pinning behavior, very much like in $\mathrm{NbSe}_{2}$, although the vortex patterns are still very different. It is particularly relevant to find out which mechanism defines the preferential orientation of vortex stripes. In the absence of extended vortex pinning centers, this feature can be hardly described by only taking into account a weak diluted pinning landscape. Furthermore, $\mathrm{MgB}_{2}$ is usually considered as a twoband material having an anisotropic $s$-wave pairing potential with uniaxial symmetry superconductor, ${ }^{24}$ which rules out the possibility that in-plane modulations of the order parameters could cause some preferential vortex stripe orientations. We can also rule out the pinning produced by omnipresent surface defects, such as terraces, since we have already demonstrated via Bitter decorations that the vortex stripes actually run across through terraces without being aligned by them. ${ }^{3}$ In turn, such modulations can be distinguished by the STM tip at the Hall sensor during the SHPM experiments. Since we did not detect these surface modulations, we can rule out terraces as a possible source of the vortex stripes. It is worth mentioning that, using the magneto-optical technique, Soibel et al. ${ }^{25}$ observed inhomogeneous flux distributions in $\mathrm{Bi}_{2} \mathrm{Sr}_{2} \mathrm{CaCu}_{2} \mathrm{O}_{8}$ single crystals grown by the floating-zone method. These unusual flux patterns persisted up to magnetic fields as high as $100 \mathrm{Oe}$, and they were related to compositional inhomogeneities of the crystal and to structural defects. We can certainly rule out this effect in our $\mathrm{MgB}_{2}$ single crystals, where the unusual patterns are observed only up to fields as high as 7-10 Oe. ${ }^{3}$ At such fields, the first neighbor distance $d_{v v}$ for a regular type- 2 system is of $1.83-1.54 \mu \mathrm{m}$, and when $d_{\min }>$ $d_{v v}$, we find the whole surface covered by a vortex lattice, even though the interaction is of type 1.5 . $^{1,12}$

In order to investigate whether the orientation of the vortex stripes responds to a particular sample boundary effect, we took SHPM images at the sample's border. Figure 5(a) shows FCs at +3 Oe taken close to the sample's edge (indicated by a red/dark gray line) at $T / T_{c}=t=0.78$. The picture shows a vortex-free region close to the edge of a length ranging between $\sim 10-20 \mu \mathrm{m}$. Similar vortex-free regions close to the edge have been observed in $\mathrm{NbSe}_{2}$ and were attributed to geometrical barrier effects. ${ }^{26,27}$ Behind the vortex-free region, vortices nucleate and form clusters with a tendency to be aligned parallel to the sample's edge. However, Fig. 5(b), corresponding to an $\mathrm{FC}$ at $-3 \mathrm{Oe}$ and in a different spot close to the sample's border, shows that the stripes form a certain angle with respect to the sample border. This behavior suggests that there is no straightforward correlation between the border of the sample and the orientation of the observed vortex stripes.

The stability of the vortex stripes in $\mathrm{MgB}_{2}$ can be investigated by applying an ac shaking. It is well known that, in the presence of a weak disorder (i.e. weakly distorted vortex lattices), vortices can be reordered by a symmetric shaking of the magnetic field. ${ }^{28}$ In that sense, one may wonder if a similar disorder-to-order transition can be induced in $\mathrm{MgB}_{2}$. Starting from an FC at -3 Oe of the $\mathrm{MgB}_{2}$ crystal taken at $t=0.78$ as shown in Fig. 5(b), we then shake the lattice at $t \sim 0.9$ by applying an ac magnetic field of 10 Oe oscillating at a frequency of $77.1 \mathrm{~Hz}$. The result (after switching off the ac field and cooling down back to $t=0.78$ ) is shown in Fig. 5(c). Due to the strong shaking, vortices as far as $95 \mu \mathrm{m}$ away from the edge (left-most vortices) have been displaced from their initial positions, but contrary to what has been observed in low pinning type-2 superconductors, ${ }^{29}$ no disorder-to-order transition is observed. Moreover, vortex clusters and stripes persist, and they have realigned themselves following a different orientation. Additionally, after the shaking [Fig. 5(c)], vortices inside stripes show the tendency to have moved closer together, as revealed by the clear darkening and difficulty to further distinguish individual vortices at some spots. This reveals that $d_{\text {min }}$ may decrease with decreasing temperature, which is in agreement with the landscape of competing $v-v$ interactions proposed by the theory of 1.5 superconductivity, where $d_{\min } \sim 1.25 \mu \mathrm{m}$ in $\mathrm{MgB}_{2}$ for the vortex lattice freezing at $t_{q}=0.9 .^{12}$ Once more, this clearly demonstrates that pinning cannot account for the observed inhomogeneous vortex patterns, like vortex stripes, chains, and 


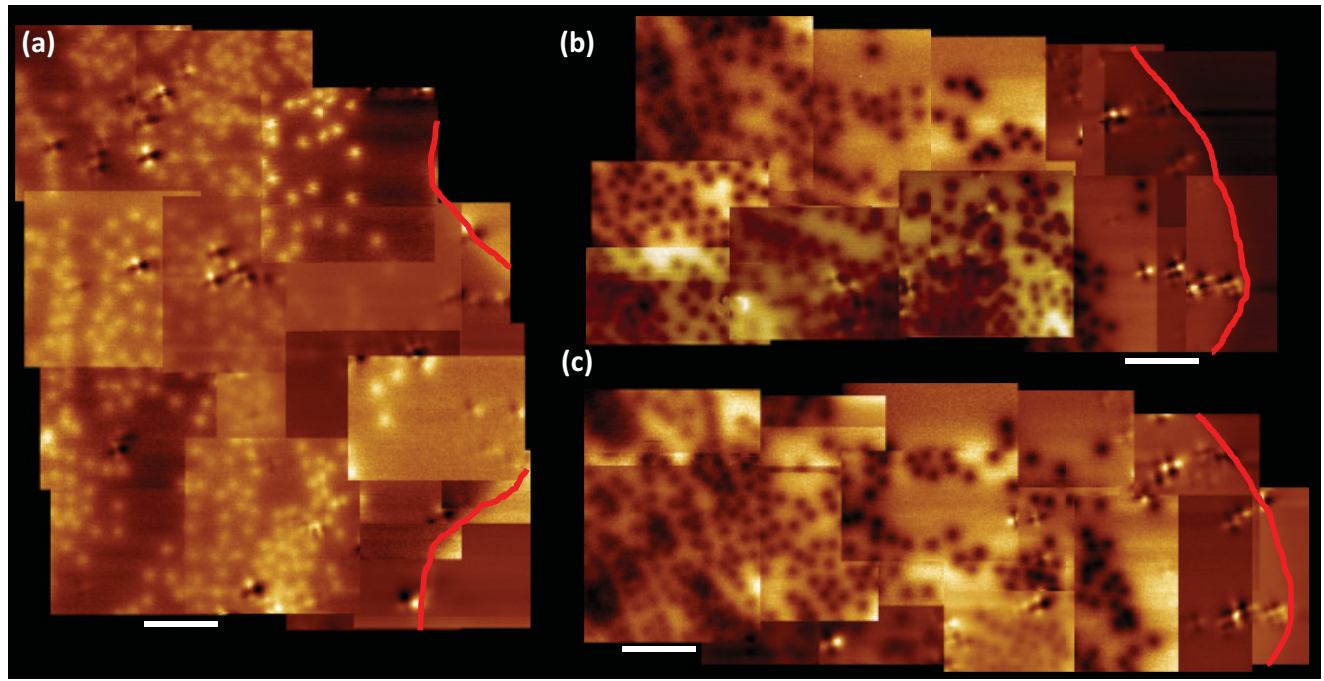

FIG. 5. (Color online) (a) Field cooling at +3 Oe for the $\mathrm{MgB}_{2}$ crystals close to the sample's border (highlighted in red/dark gray). (b) Field cooling at -3 Oe for the $\mathrm{MgB}_{2}$ crystal, and (c) image of the same region after shacking the vortex lattice at $t=0.9$ and with and $H_{a c}=10 \mathrm{Oe}, f=77.1 \mathrm{~Hz}$. The images are taken at $30 \mathrm{~K}$. Pictures are at the same scale, the white bar below each picture indicates $10 \mu \mathrm{m}$.

clusters, and more likely they are the result of competing $v-v$ interactions.

\section{CONCLUSIONS}

By directly visualizing the vortex patterns via scanning Hall probe microscopy, we have provided convincing direct experimental evidence that the anomalous vortex distributions observed in $\mathrm{MgB}_{2}$ are not caused by inhomogeneous pinning landscapes, even though pinning or surface barriers are playing an important role in trapping the vortices at fields much lower than the lower critical field. These results further support the application of the type-1.5 superconductivity scenario to clean crystals of the two-gap $\mathrm{MgB}_{2}$ superconductor. In addition, we have tracked the evolution of the different patterns as a function of the vortex density $(B)$ and interaction strength $(T)$; as the density increases, the system progresses from a low-density clump phase to an intermediate-density stripe phase and then to a higher-density stripe phase, where organized voids appear in the system, thus showing that 1.5 superconductors are a good landscape to study collections of particles with competing interactions. It is important to stress that all theoretical studies concerning unconventional vortex-vortex interactions performed so far do not include vortex pinning. ${ }^{3,12,30}$ Further studies including this factor may be crucial to obtain a more complete picture of the dominant mechanism determining the vortex pattern formation in twoband superconductors, as pointed out by molecular dynamic simulations in systems with competing short- and long-range interactions. $^{20}$

\section{ACKNOWLEDGMENTS}

This work was supported by Methusalem funding by the Flemish government and the Flemish Science Foundation, and by the Fund for Scientific Research - projects G.0370.09N and G.0115.12N. J.G. and A.V.S. acknowledge support from FWO-Vl. *joffre.gutierrezroyo@fys.kuleuven.be

${ }^{1}$ M. Seul and D. Andelman, Science 267, 476 (1995).

${ }^{2}$ M. Klokkenburg, R. P. A. Dullens, W. K. Kegel, B. H. Erné, and

A. P. Philipse, Phys. Rev. Lett. 96, 037203 (2006).

${ }^{3}$ V. V. Moshchalkov, M. Menghini, T. Nishio, Q. H. Chen, A. V. Silhanek, V. H. Dao, L. F. Chibotaru, N. D. Zhigadlo, and J. Karpinski, Phys. Rev. Lett. 102, 117001 (2009).

${ }^{4}$ H. London, Proc. R. Soc. London A 152, 650 (1935).

${ }^{5}$ V. L. Ginzburg and L. D. Landau, Zh. Eksp. Teor. Fiz. 20, 1064 (1950).

${ }^{6}$ E. Babaev and M. J. Speight, Phys. Rev. B 72, 180502 (2005).

${ }^{7}$ T. Nishio, V. H. Dao, Q. Chen, L. F. Chibotaru, K. Kadowaki, and Victor V. Moshchalkov, Phys. Rev. B 81, 020506(R) (2010).

${ }^{8}$ J. Karpinski, N. D. Zhigadlo, S. Katrych, R. Puzniak, K. Rogacki, and R. Gonnelli, Physica C 456, 3 (2007).
${ }^{9}$ S. M. Kazakov, R. Puzniak, K. Rogacki, A. V. Mironov, N. D. Zhigadlo, J. Jun, Ch. Soltmann, B. Batlogg, and J. Karpinski, Phys. Rev. B 71, 024533 (2005).

${ }^{10}$ M. Zehetmayer, M. Eisterer, J. Jun, S. M. Kazakov, J. Karpinski, A. Wisniewski, and H. W. Weber, Phys. Rev B 66, 052505 (2002).

${ }^{11}$ L. Li, Z. Xu, J. Shen, L. Qiu, and Z. Gan, J. Phys.: Condens. Matter 17, 493 (2005)

${ }^{12}$ A. Levchenko, M. R. Norman, and A. A. Varlamov, Phys. Rev. B 83, 020506(R) (2011).

${ }^{13}$ E. Zeldov, A. I. Larkin, V. B. Geshkenbein, M. Konczykowski, D. Majer, B. Khaykovich, V. M. Vinokur, and H. Shtrikman, Phys. Rev. Lett. 73, 1428 (1994).

${ }^{14}$ M. Marchevsky, P. H. Kes, and J. Aarts, Physica C 282-287, 2083 (1997). 
${ }^{15}$ R. B. G. Kramer, G. W. Ataklti, V. V. Moshchalkov, and A. V. Silhanek, Phys. Rev. B 81, 144508 (2010).

${ }^{16}$ I. V. Grigorieva, Supercond. Sci. Technol. 7, 161 (1994).

${ }^{17}$ A. I. Larkin and Y. Ovchinnikov, J. Low Temp. Phys. 34, 409 (1979).

${ }^{18}$ G. Karapetrov, J. Fedor, M. Iavarone, D. Rosenmann, and W. K. Kwok, Phys. Rev. Lett. 95, 167002 (2005).

${ }^{19}$ V. H. Dao, L. F. Chibotaru, T. Nishio, and V. V. Moshchalkov, Phys. Rev. B 83, 020503(R) (2011).

${ }^{20}$ C. Reichhardt, C. J. Olson Reichhardt, I. Martin, and A. R. Bishop, Phys. Rev. Lett. 90, 026401 (2003).

${ }^{21}$ H. Dai, S. Yoon, J. Liu, R. C. Budhani, and C. M. Lieber, Science 265, 1552 (1994).

${ }^{22}$ See Supplemental Material at http://link.aps.org/supplemental/ 10.1103/PhysRevB.85.094511 for animation slide 1 .

${ }^{23}$ See Supplemental Material at http://link.aps.org/supplemental/ 10.1103/PhysRevB.85.094511 for animation slide 2.

${ }^{24}$ P. Seneor, C.-T. Chen, N.-C. Yeh, R. P. Vasquez, L. D. Bell, C. U. Jung, M.-S. Park, H.-J. Kim, W. N. Kang, and S.-I. Lee, Phys. Rev. B 65, 012505 (2001).
${ }^{25}$ A. Soibel, Y. Myasoedov, M. L. Rappaport, T. Tamegai, S. S. Banerjee, and E. Zeldov, Phys. Rev. Lett. 87, 167001 (2001).

${ }^{26}$ M. Marchevsky, L. A. Gurevich, P. H. Kes, and J. Aarts, Phys. Rev. Lett. 75, 2400 (1995).

${ }^{27}$ A. A. F. Olsen, H. Hauglin, T. H. Johansen, P. E. Goa, and D. Shantsev, Physica C 408-410, 537 (2004).

${ }^{28}$ S. O. Valenzuela and V. Bekeris, Phys. Rev. Lett. 86, 504 (2001).

${ }^{29}$ N. Avraham, B. Khaykovich, Y. Myasoedov, M. Rappaport, H. Shtrikman, D. E. Feldman, T. Tamegai, P. H. Kes, M. Li, M. Konczykowski, K. van der Beek, and E. Zeldov, Nature 411, 451 (2001).

${ }^{30}$ E. Babaev, J. Carlström, and M. J. Speight, Phys. Rev. Lett. 105, 067003 (2010); J. Carlström, E. Babaev, and M. J. Speight, Phys. Rev. B 83, 174509 (2011); M. Silaev and E. Babaev, ibid. 84, 094515 (2011); E. H. Brandt, R. G. Mints, and I. B Snapiro, Phys. Rev. Lett. 76, 827 (1996); G. Blatter, and V. B. Geshkenbein, ibid. 77, 4958 (1996); A. Jacobs, Phys. Rev B 4, 3029 (1971); A. Chaves, L. Komendová, M. V. Milosevic, J. S. Andrade, G. A. Farias, and F. M. Peeters, Phys. Rev. B 83, 214523 (2011). 